\title{
Abdomen agudo secundario a sangrado masivo por rotura de metástasis hepáticas de un tumor del estroma gastrointestinal
}

\author{
Acute abdomen secondary to massive bleeding due to rupture of liver metastases of a \\ gastrointestinal stroma tumor
}

Julio Santoyo-Villalba*, Jennifer Triguero-Cabrera y Alejandro García-Jiménez

Servicio de Cirugía General y Aparato Digestivo, Hospital Universitario Virgen de las Nieves, Granada, España

\begin{abstract}
Resumen
El hemoperitoneo secundario a estallido hepático no traumático es una causa poco frecuente de abdomen agudo. Se presenta el caso de una paciente con estallido hepático secundario a metástasis de un tumor del estroma gastrointestinal (GIST) que requirió una laparotomía emergente por inestabilidad hemodinámica. Se evidenció intraoperatoriamente una lesión de más de $20 \mathrm{~cm}$ dependiente de la pared posterior gástrica y un hemoperitoneo secundario a estallido de metástasis hepáticas y descapsulación de la cápsula de Glisson. Una complicación frecuente de los GIST es la rotura que ocasiona hemorragia digestiva o hemoperitoneo, como ocurrió en nuestro caso.
\end{abstract}

Palabras clave: Hemoperitoneo. Tumores del estroma gastrointestinal. Metástasis.

\begin{abstract}
Hemoperitoneum secondary to non-traumatic liver rupture is a rare cause of an acute abdomen. We present the case of a patient with a hepatic rupture secondary to metastasis of a gastrointestinal stromal tumor (GIST) who required an emergent laparotomy due to hemodynamic instability. Intraoperatively, a lesion of more than $20 \mathrm{~cm}$ dependent on the posterior gastric wall and a hemoperitoneum secondary to rupture of liver metastases and decapsulation of the Glisson capsule was evidenced. A frequent complication of GIST tumors is its rupture causing gastrointestinal bleeding or hemoperitoneum, as occurred in our case.
\end{abstract}

Key words: Hemoperitoneum. Gastrointestinal stromal tumors. Metastasis.

\section{Introducción}

Los tumores del estroma gastrointestinal (GIST) son los tumores mesenquimales más frecuentes y se caracterizan por la expresión de un receptor de la tirosina cinasa, el c-KIT/CD 1171. Hasta el $60 \%$ se originan en el estómago ${ }^{2}$. Los síntomas de presentación más frecuentes son sangrado en la cavidad peritoneal, hemorragia digestiva y anemia, entre otros. Aproximadamente un $20 \%$ de los GIST presentan metástasis en el momento del diagnóstico, normalmente peritoneales y hepáticas. El tratamiento de los GIST metastásicos es el imatinib, que compite con el ATP en la fijación del dominio de la tirosina

\footnotetext{
Correspondencia:

*Julio Santoyo-Villalba

Avda. de las Fuerzas Armadas 2

C.P. 18014, Granada, España

E-mail: jsantoyovil@gmail.com

Fecha de recepción: 27-11-2020

Fecha de aceptación: 29-01-2021

DOI: 10.24875/CIRU.20001313

Cir Cir. 2021;89(S1):93-96 Contents available at PubMed www.cirugiaycirujanos.com 0009-7411/@ 2021 Academia Mexicana de Cirugía. Publicado por Permanyer. Este es un artículo open access bajo la licencia CC BY-NC-ND (http://creativecommons.org/licenses/by-nc-nd/4.0/).
} 
cinasa e inhibe la activación y la fosforilación del mis$\mathrm{mo}^{3}$. La duración del tratamiento debe ser de por vida. No obstante, cuando existe una complicación aguda, como el sangrado a la cavidad abdominal, el tratamiento en ocasiones debe ser quirúrgico, sobre todo en casos de inestabilidad hemodinámica en los que otros procedimientos no deben llevarse a cabo.

Existen muy pocos casos publicados en la literatura en que el hemoperitoneo sea secundario a la rotura de metástasis hepáticas, como ocurrió en la paciente de nuestro caso que exponemos a continuación.

\section{Caso clínico}

Mujer de 38 años sin antecedentes de interés que acudió a urgencias por dolor abdominal en el epigastrio de varios meses de evolución, con empeoramiento súbito en las últimas horas. No había presentado náuseas, vómitos, fiebre, molestias urinarias, distensión abdominal ni alteración del tránsito intestinal. A su llegada a urgencias tuvo varios episodios eméticos de contenido bilioso y asoció cortejo vegetativo con sudoración profusa. En la exploración física se evidenció un abdomen blando, con molestia a la palpación en el epigastrio, donde se apreciaba una tumoración pétrea. No existían signos de irritación peritoneal generalizada. Hemodinámicamente se encontraba estable. En la analítica destacaban hemoglobina de $12.5 \mathrm{~g} / \mathrm{dl}$ y lactato deshidrogenasa de 701 , siendo el resto anodino. Se solicitó una ecografía de abdomen (Fig. 1) que evidenció una gran masa gástrica y lesiones hepáticas bilobares sugerentes, en primer lugar, de metástasis. Durante la realización de la ecografía la paciente presentó un cuadro de hipotensión arterial, taquicardia y disminución progresiva del nivel de consciencia. Se realizó una gasometría venosa urgente que evidenció acidosis láctica y hemoglobina de $7.5 \mathrm{~g} / \mathrm{dl}$ (anemización de 5 puntos en 1 hora). Ante la sospecha de un sangrado agudo e inestabilidad hemodinámica, se trasladó a la paciente a quirófano para cirugía emergente. Se realizó laparotomía media y se evidenció una masa tumoral de más de $20 \mathrm{~cm}$ (Fig. 2) que parecía depender de la pared posterior gástrica, alojada en la transcavidad de los epiplones, con un hemoperitoneo masivo asociado de más de 2 litros secundario al estallido de las metástasis hepáticas y la descapsulación de toda la cápsula de Glisson del hígado derecho. Así mismo, se evidenció una carcinomatosis peritoneal extensa, de la que se tomaron biopsias. Se realizó resección

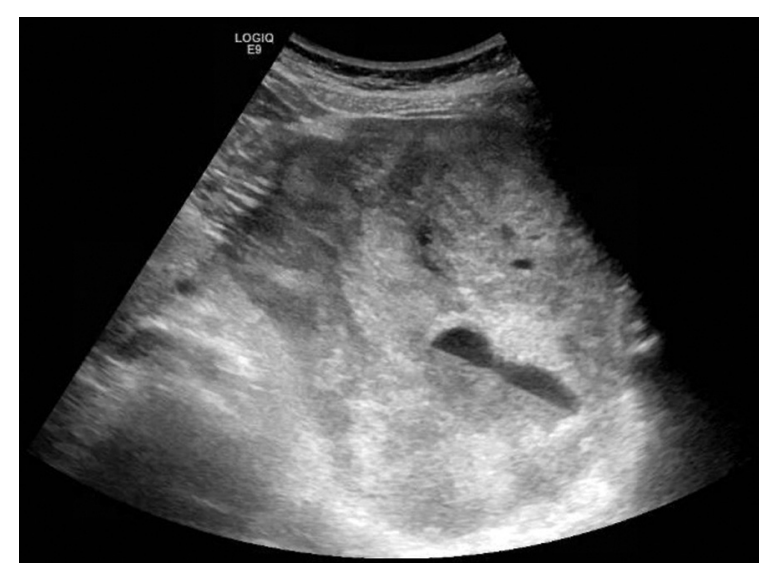

Figura 1. Ecografía en la que se evidencia gran masa gástrica sugerente de GIST.

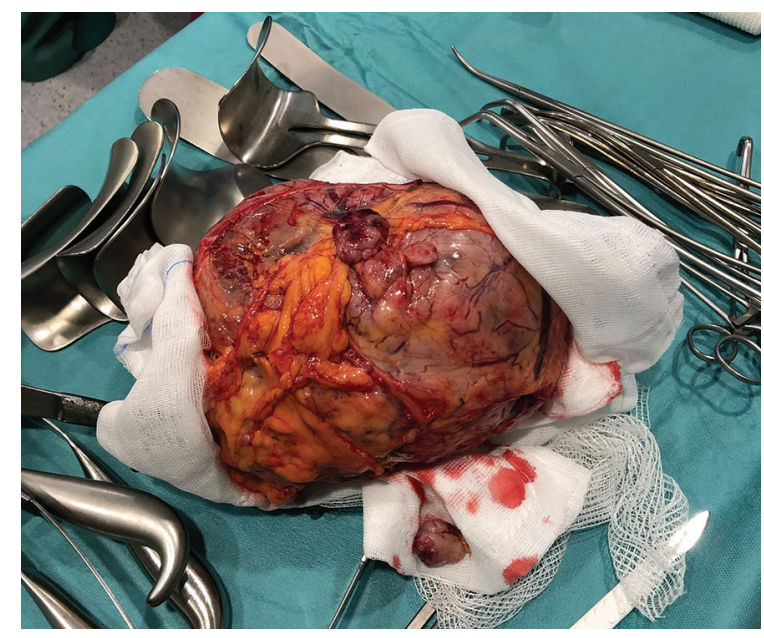

Figura 2. Pieza quirúrgica.

de la tumoración gástrica, precisando una resección atípica de la curvatura mayor.

Debido a la extensión del sangrado del hígado, se realizó packing hepático con múltiples compresas, dejando el abdomen abierto con un sistema de terapia de presión negativa tipo VAC. En los días posteriores se revisó hasta en dos ocasiones en quirófano para recambio y retirada, respectivamente, de dicho packing una vez comprobado el cese de la hemorragia. La evolución posterior en la sala de hospitalización fue favorable y se le dio el alta el día 24 de posoperatorio, sin mayores complicaciones médicas ni quirúrgicas. En la figura 3 se pueden ver las lesiones hepáticas que presentaba la paciente, evidenciadas en una tomografía computarizada (TC) realizada tras el alta hospitalaria. La anatomía patológica 


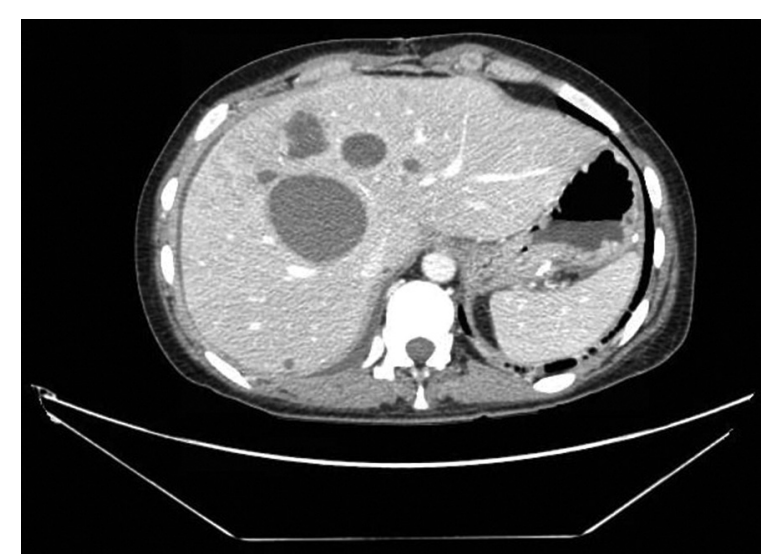

Figura 3. Tomografía computarizada en la que se evidencian múltiples metástasis hepáticas.

informó de un GIST de grado 1 y pT4 según la clasificación TNM. Asimismo, las células tumorales fueron positivas para DOG1, CD117 y actina de músculo liso (débil y focal), y negativas para desmina y CKAE1/ AE3. Los implantes evidenciados (hepáticos y mesentéricos, de los cuales se obtuvieron biopsias) fueron compatibles con metástasis de GIST («múltiples implantes de tumor estromal gastrointestinal de células fusiformes»), confirmando el estadio M1 del tumor. Por este motivo, la paciente estuvo en tratamiento con sunitinib durante 1 año y se logró una remisión completa evidenciada por TC. Como efecto adversó desarrolló una trombosis de la vena cava inferior, ambas venas iliacas comunes y externas, y una trombosis distal de la vena suprahepática media, tratadas con éxito con heparina a dosis anticoagulantes.

\section{Discusión}

Los GIST son los tumores mesenquimales más frecuentes. Hasta el $60 \%$ se originan en el estómago, como ocurrió en nuestro caso, aunque también pueden aparecer en otros órganos, como el intestino delgado $(20-25 \%)$, el colon y el recto $(5 \%)$, o el esófago $(5 \%)$, e incluso en localizaciones extraintestinales como el mesenterio, el epiplón y el peritoneo. La presentación clínica dependerá de la localización y del tamaño del tumor; a menudo son asintomáticos o presentan sintomatología inespecífica, como dolor abdominal, dispepsia o distensión abdominal.

Los GIST pueden diferenciarse de otros tumores mesenquimales, como el leiomioma y el leiomiosarcoma, por su expresión génica. Más del $95 \%$ de los GIST expresan CD117, o c-kit, un receptor de la tirosina cinasa. Esto permite que para su tratamiento se usen inhibidores de la tirosina cinasa, como el imatinib.

En ocasiones pueden debutar con cuadros abdominales agudos, como obstrucción intestinal o sangrado digestivo ${ }^{4}$. El tratamiento de los tumores localizados suele ser quirúrgico y en general no es necesario realizar linfadenectomía debido a la baja probabilidad de invasión linfática que presentan este tipo de tumores.

Una complicación importante de estos tumores, cuando no se diagnostican en una etapa temprana, es su rotura y en consecuencia hemorragia digestiva o hemoperitone $0^{5}$. Las características de los GIST que ocasionan sangrados (hemorragia digestiva o hemoperitoneo) incluyen anatomía patología de alto riesgo, gran tamaño (promedio de $10 \mathrm{~cm}$ ), crecimiento exofítico, necrosis interna o degeneración quística, y crecimiento rápido ${ }^{6}$.

El lugar más frecuente de metástasis es el hígado. Estas metástasis hepáticas pueden ocurrir incluso más de 30 años después del diagnóstico del tumor primario ${ }^{7}$. Es sumamente infrecuente, pero se han descrito algunos casos de hemoperitoneo por rotura de metástasis hepáticas, como ocurrió en nuestro caso. El tratamiento de las complicaciones agudas de las metástasis de estos tumores en ocasiones es quirúrgico.

La rotura de estos tumores incrementa sustancialmente la probabilidad de diseminación peritoneal, tal como sucedió en esta paciente ${ }^{8}$.

Debido al variable intervalo hasta la recurrencia de este tipo de tumores, es necesario determinar el periodo de seguimiento necesario de los GIST tras la cirugía. La guía japonesa de GIST recomienda el seguimiento durante los 10 años posteriores a la cirugía del tumor primario ${ }^{9}$.

\section{Agradecimientos}

A todos los miembros del servicio de cirugía general y del aparato digestivo del Hospital Universitario Virgen de las Nieves, de Granada (España), y a todo el equipo de enfermería y anestesiología que estuvo presente en el acto quirúrgico.

\section{Responsabilidades éticas}

Protección de personas y animales. Los autores declaran que para esta investigación no se han realizado experimentos en seres humanos ni en animales. 
Confidencialidad de los datos. Los autores declaran que han seguido los protocolos de su centro de trabajo sobre la publicación de datos de pacientes.

Derecho a la privacidad y consentimiento informado. Los autores han obtenido el consentimiento informado de los pacientes y/o sujetos referidos en el artículo. Este documento obra en poder del autor de correspondencia.

\section{Financiamiento}

Los autores declaran que no se recibió ningún financiamiento para la realización de este trabajo.

\section{Conflicto de intereses}

Los autores declaran que no existen conflicto de intereses.

\section{Bibliografía}

1. Eizaguirre B, Burgos JJ. Tumores GIST. Revisión de la literatura. Rev Esp Patol. 2006;39:209-18.

2. Martín R, García J. Tumor del estroma gastrointestinal (GIST). Rev Esp Enferm Dig. 2014;106:227.

3. Buchdunger E, O'Reilly T, Wood J. Pharmacology of imatinib (STI571). Eur J Cancer. 2002;38 (Suppl 5):S28-S36.

4. Fernández LI, Álvarez T, Sanz A, Velayos B, Aller R, González JM. Tumores de la estroma gastrointestinal (GIST): aspectos clínicos. Rev Esp Enferm Dig. 2007;99:19-24.

5. Kim TH, Choi SC, Choi CS, Nah YH. Hemoperitoneum secondary to a ruptured gastric stromal tumor. Gastrointest Endosc. 2006;63:1066-7.

6. Kim SW, Kim HC, Yang DM. Perforated tumours in the gastrointestinal tract: CT findings and clinical implications. Br J Radiol. 2012;85: 1307-13.

7. Ishizaki M, Uno F, Yoshida R, Miyauchi S, Honda O. Very delayed liver metastasis from small bowel gastrointestinal stromal tumor (32 years after resection of the small bowel GIST): report of a case. Int J Surg Case Rep. 2020;76:156-60.

8. Shively J, Ebersbacher C, Walsh WT, Allemang MT. Spontaneous hemoperitoneum from a ruptured gastrointestinal stromal tumor. Cureus. 2020;12:e9338.

9. Nishida T, Hirota S, Yanagisawa A, Sugino Y, Minami M, Yamamura Y, et al. Clinical practice guidelines for gastrointestinal stromal tumor (GIST) in Japan: English version. Int J Clin Oncol. 2008;13:416-30. 